\title{
RESOLUTION ENHANCEMENT OF ELECTRON MICROSCOPIC VOLUME BY VOLUME RESTORATION TECHNIQUE
}

\author{
Anik Khan ${ }^{1}$, Kishor Datta Gupta ${ }^{1}$, and Ariful Haque ${ }^{2}$ \\ ${ }^{1}$ Department of Computer Science, University Of Memphis , Memphis,TN, \\ USA,3811 \\ ${ }^{2}$ Department of Electrical and Electronic Engineering, Bangladesh University \\ of Engineering and Technology, Dhaka-1205
}

\begin{abstract}
The knowledge of structure of proteins, protein derived compounds and RNA structures in eukaryotic cell is mandatory to understand the functions of these macromolecules. With recent development of Direct Electron Detector Device (DDD) camera and application of maximum likelihood algorithms in volume reconstruction,cryo Electron Microscopy (cryo-EM) enables us to visualize the macromolecules in nearly physiological state. The current resolution limit of cryo-EM can be improved further by applying novel and effective signal processing algorithms after the EM workflow. In this work, a signal processing method has been developed to enhance the resolution of the EM volume through volume restoration techniques. We have proposed a novel technique to estimate the volume degradation function of the volume reconstruction system from the noise-only subvolumes of the observed EM volume. Then the volume is restored (utilizing the estimated volume degradation function) using a combination of regularized Richardson-Lucy and Wiener Deconvolution techniques. In addition to volume restoration, we have employed spatial de-noising techniques utilizing various morphological filters to reduce noise outside the main molecular structure. The experimental results demonstrate that the resolution (evaluated by ourier Shell Correlation curve) and visual quality of the EM volume can be significantly improved using our proposed technique.
\end{abstract}

\section{KEYWORDS}

Electron Microscopic Signal processing Visualization volume degradation function Image processing

\section{INTRODUCTION}

Thousands of different types of functions are performed in eukaryotic cell by protein and protein derived complexes. The shape and transformation of these complexes infer about the funtions performed by these macromolecules. As a result, 3D structure of these macromolecules are the main study of structural genomics [1]. So, precise structural information of macromolecules in eukaryotic cell is of great value.

X-ray crystallography and Nuclear Magnetic Resonance (NMR) are two most common experimental techniques to achieve high-resolution structure of macromolecules. Standard resolution for X-ray and NMR ranges between $1.5 \AA$ and $3 \AA$. As crystallization is needed for Xray diffraction and large macromolecules cannot be resolved by NMR, all macromolecules are not amenable to X-ray and NMR. In addition, these two techniques require relatively high macromolecular concentration. Besides, one of the aims of structural genomics is to cut down the

Dhinaharan Nagamalai et al. (Eds) : SAI, NCO, SOFT, ICAITA, CDKP, CMC, SIGNAL - 2019 pp. 131-143, 2019. () CS \& IT-CSCP 2019

DOI: $10.5121 /$ csit.2019.90710 
cost of macromolecular structure determination. Electron Microscopy (EM) is an alternative structure determination technique (Experimental) that resolves the limitations of X-ray and NMR.

EM is capable of looking at structures in nearly physiological state, it does not require high concentrations and can naturally handle large macromolecules. However, the resolution of EM is rather low compared to X-ray crystallization and NMR techniques. The low resolution of volume reconstructed from the electron microscope is due to several reasons ([2] [3]) such as the loss of overall contrast by the EM, abberration at high frequencies, conformational variability of the molecules, different types of beam-induced motions ([4][5]). Ultimately, the macromolecule is not isolated in the void. It is girded by a light coating of amorphous ice (cryo-EM) or a concentrated layer of carbon and a heavy metal salt depending on the sample preparation method. As a result, the specimen under study offers a vital background signal. Collectively, the Signal-toNoise Ratio (SNR) becomes under 0.1.[6] [3].

In the last decade, significant achievements have attained wide attention of the community to cryo-EM. The introduction of Direct Electron Detector device (DDD) camera [7] and Maximum Likelihood algorithms into cryo-EM has made macromolecules visible nearly at physiological state. High detective quantum efficiency (DQE) [8] and underlying CMOS (complementary metal-oxide semiconductor) technology has enabled the production of high quality micrograph. The inevitable small movements due to the electron beam can be compensated because of fast readout of DDD cameras. In addition, continually increasing computational power allows the use of increasingly sophisticated image processing algorithms, resulting in greatly improved resolution and more reliable 3D density maps [9]. As a result of all these developments, near atomic resolution $(<4)$ has been achieved and this resolution improvement is refered to as "Resolution Revolution in cryo-EM". But still, X-ray will be a better choice for any small (below $100 \mathrm{kD}$ ) macromolecule structure to have $2 \AA$ or better resolution.

The current electron microscopy (EM) resolution limit ( $4 \AA$ to $3 \AA$ (with a world record of $2.2 \AA$ [11]) [3] [12][13] can be pushed forward by applying volume restoration techniques already developed for other signal processing applications. Volume restoration is an inverse process whose objective is to recover a high resolution (HR) structure from a low resolution (LR) degraded structure by retriving structural information. It is to be mentioned here that these signal processing algorithms will be applied after the complete workflow of electron microscopy procedure (from micrograph screening to $3 \mathrm{D}$ reconstruction including contrast transfer function correction).

In this paper, we propose a novel methodology to improve the resolution of EM volume by volume restoration technique. First, an estimation of volume degradation function of EM volume reconstruction system is estimated from 3D reconstructed volume available after EM volume reconstruction workflow. The background noise outside the main molecular structure are utilized for this purpose. This outside noise of main molecular structure can be modeled as the convolution of the input noise from the amorphous ice and the volume degradation function. As a result, the estimation of volume degradation function can be estimated from the magnitude spectrum of the outside noise data. Second, after estimating the estimation of volume degradation function, we use regularized Richardson-Lucy deconvolution technique [14] [15] and Wiener deconvolution [16] to restore the HR volume. The regularization ensures stable performance during deconvolution operation. Finally, some post-processing operations further improves the SNR and visual quality of the restored HR volume. Simulation results verify the effectiveness of the proposed technique for EM resolution enhancement. 
The rest of the paper is organized as follows. Section II breifly states the workflow of 3D reconstruction in Electron Microscopy. Section III presents the proposed volume reconstruction technique. Experimental results are shown in section IV. Conclusion is given in the last section.

\section{Review OF 3D Volume Reconstruction IN Electron MicROSCOPE}

As stated earlier, our resolution enhancement module is applied on the 3D reconstructed volume available after EM volume reconstruction workflow. For the sake of completeness and better understanding of proposed method, the standard workflow of $3 \mathrm{D}$ reconstruction in EM is described briefly.

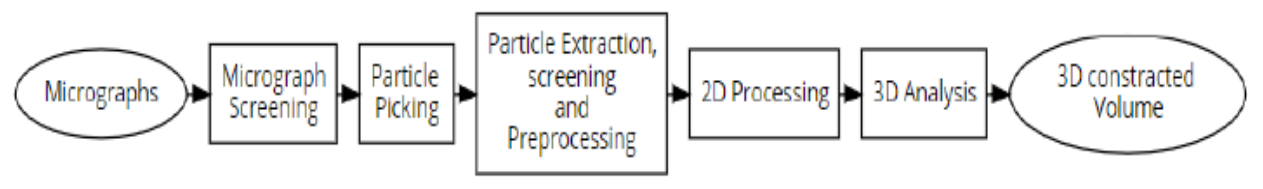

Fig. 1: EM Volume Reconstruction Workflow

The field of electron microscopy applied to structural biology has made significant progress in past eras. The main focus of this research area is to employ high-resolution electron microscopy for macromolecular structures. One of the techniques available to perform the study of macromolecules is called Single Particle Analysis (SPA) [17] [18]. SPA is a collection of image processing techniques that are used to analyze images from the Transmission Electron Microscopy (TEM). One of the techniques in SPA is to build up the three-dimensional reconstruction of the particle [17] [6]. Several software packeges are available, such as XMIPP, BSOFT, SPIDER, EMAN/EMAN2, IMAGIC, SPARX, SITUS, RELION [19] and Frealign Package [20], supporting a large variation of workflow. Among them, XMIPP provides standardized protocols from selecting micrograph to 3D reconstruction. The proposed method can be used as an extension of these software packages. A standardized flow diagram is shown in Fig. 1. The steps of the processing of Electron Microscopy for SPA are as follows :
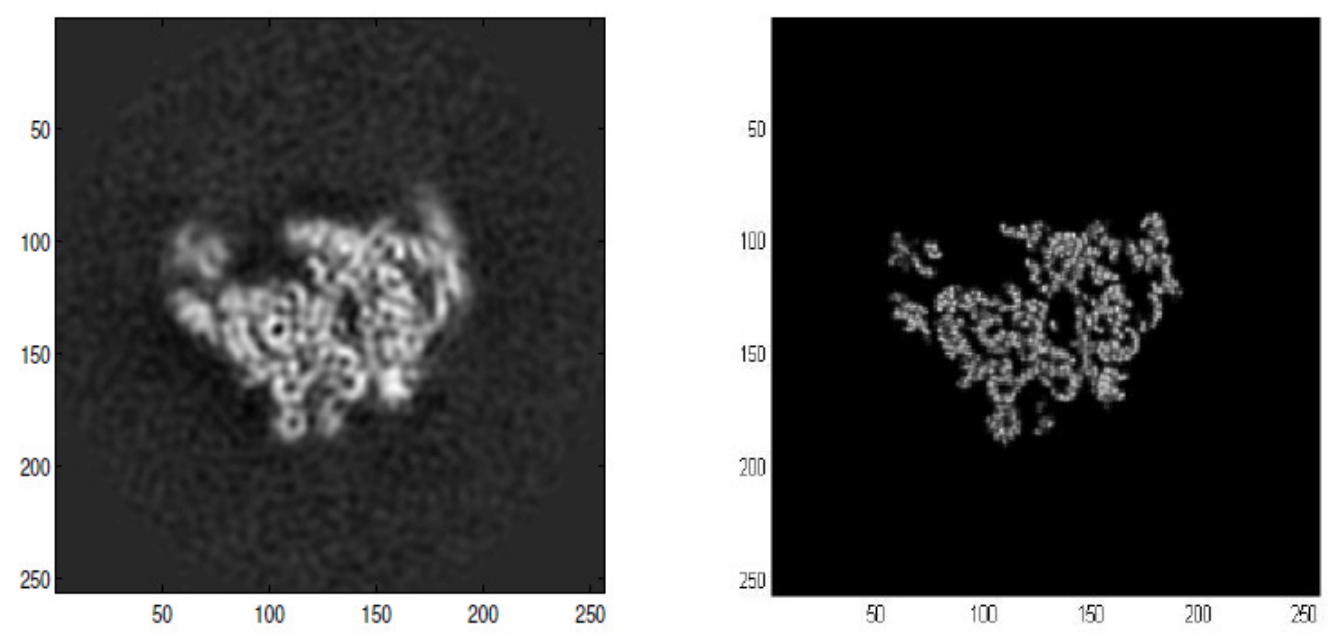

Fig. 2: (Left) Slice of a 3D reconstructed volume of 50S ribosome subunit bound with ObgE. (Right) Ground truth of the 50S ribosome subunit bound with ObgE. X and Y both represents pixel positions here 
In Micrograph Screening state, Micrographs are directly generated from TEM which are visible to naked eye. Thousands of micrograhs are produced for SPA. But all these micrographs are not suitable for further analysis. The purpose of this step is to vilidify the quality of micrographs for further processing. Only high quality micrographs should be selected for 3D reconstruction. Good micrographs have homogeneous background level, are not astigmatic, have no drift, have high resolution structural information.

In Particle Picking state, Only small portion of micrograph, consisting of particles that the user interested in, is needed for next steps. So, in this step, particle will be picked from micrographs selected from the previous screening step. The purpose of this step is to select the particles that user is interested in. Particle picking can be manual or semiautomic.

In Particle Extraction, Screening and Pre-processing step, the particles selected from the micrographs in previous step, are extracted and a stack of projections of the particles is formed for further analysis in the next steps. In Particle Extraction step, the particles selected from the previous step are extracted. When extracting particles we can take a number of actions like phase flipping, contrast inversion, normalization etc.

In particle picking step, it is possible to pick wrong particle that will deteriorate the final reconstructed volume. So, in Particle Screening step some processing is done so that the worngly picked particles are sorted at the end of a stack. At this stage, user can discard the wrongly picked particles, particles on edges and contaminants. In Particle Preprocessing step [44], user can use image processing filter to reduce [43], highlight certain features, mask out the backgound, etc.

The main purpose of 2D Processing step is to select groups of images that are good reprensentative of the particle. In order to do that, the whole set of images is classified into different groups as homogeneous as possible. There are several algorithms for homogeneous classification. The algorithms offered in XMIPP are clustering in 2D (CL2D) [21], Maximum Likelihood in 2D (ML2D)[22], Kernel Density Estimation Self-Organizing Map (KenDenSOM) [23] and Rotational Spectra [24]. The projections that come from wrongly picked particles and contaminants will form classes different from the particle under study. The classes that are good representative of the particle will be used in $3 \mathrm{D}$ analysis.

In 3D Analysis step, we get 3D volume from a set of projections. There are two types of 3D analysis. They are - (i) Homogeneous Population Analysis (ii) Heterogeneous Population Analysis In homogeneous analysis, all the projections are assumed to be generated from same particle. We take an initial guess and search for perfect alingment of a projection. In this way, we iteratively construct the 3D volume of EMp article. During this alignment and reconstruction process, user can correct the amplitude effect of Contrast Transfer Function (CTF) using Wiener filter [25].

It is always possible that the projections belong to different molecular structures. So, each homogeneous group of projections is used to produce single 3D reconstructed volume.

The resolution of the 3D reconstructed volume (even after CTF correction) is not satisfactory enough. Volume processing techniques can be introduced on the observed volume to enhance structural resolution. 


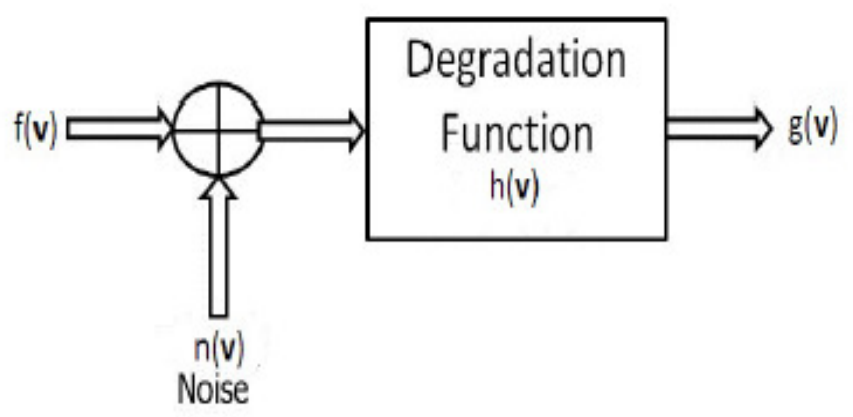

Fig. 3: Schematic diagram of volume degradation process in electron microscopy

\section{The Proposed Volume Restoration TeChNique}

Fig. 2 (Left) presents a 2D slice of 3D reconstructed volume produced from aforementioned EM workflow. Identical 2D slice of the ground truth volume ( obtained from [26] ) is shown Fig. 2 (Right) for the purpose of comparison. It is observed that the macromolecule still contains significant amout of blurring. So, there is still scope of resolution improvement by volume restoration technique. Moreover, there is a substantial amout of noise outside of macromolecule. This is due to the fact that macromolecules have to be embedded in amorphous ice at liquid nitrogen temperature(about-200 C) [27] [28] [29] to be visualized in the Electron Microscope. As a result, the acquired images contain background signal from the ice that is observed as noise. This noise undergoes identical processing as the macromolecule by EM 3D reconstruction workflow. So the macromolecular volume and noise-only sub-volume is blurred by same degradation function. We utilized this cue to estimate the volume degradation function from the noise-only sub-volumes.

The proposed technique consists of three steps: Estimation of volume degradation function from the observed volume, regularized deconvolution and post processing for automatic spatial noise reduction outside of main molecular structure.

\subsection{ESTIMATION OF VOLUME DEgRADATION FUNCTION}

The volume degradation process in Electron Microscopy can be modeled as depicted in Fig. 3. Therefore, the blurring in the observed volume can be expressed as

$$
g(\mathbf{v})=(f(\mathbf{v})+n(\mathbf{v})) * h(\mathbf{v})
$$

where $g(v), f(v)$ and $n(v)$ denote, respectively, the LR observed volume, the HR input volume and the input noise. Here, $h(v)$ is the degradation function that relates the input and output volume. Here, $v=(x, y, z)$ represents position of a voxel of a macromolecular volume and "*" denotes 3D convolution operation. In what follows, we describe the technique of estimating $h(v)$ from noiseonly sub-volumes of $\mathrm{g}(\mathrm{v})$.

Let, we haveM number of noise-only subvolumes and $\mathrm{g}_{\mathrm{i}}(\mathrm{v})$ represents the ith noise-only subvolume of observed EM volume. According to (1), gi(v) can be modeled as

$$
g_{i}(\mathbf{v})=n_{i}(\mathbf{v}) * h(\mathbf{v})
$$


where $n_{i}(v)$ is the input noise corresponding to the ith noise-only subvolume. In the frequency domain, the (2) can be written as

$$
G_{i}(\omega)=N_{i}(\omega) H(\omega)
$$
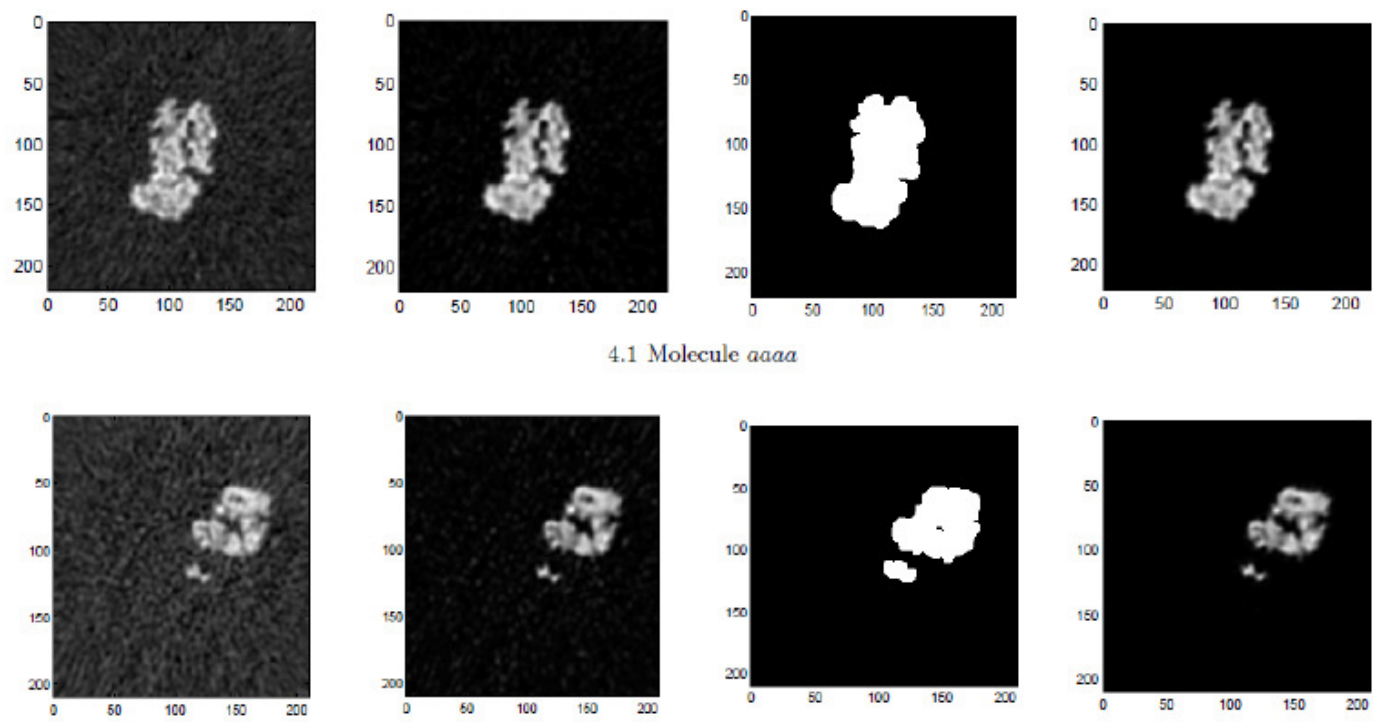

4.2 Molecule $b b b b$

Fig. 4: Images obtained at different stages of the proposed technique. (Left to right) (a) Given low resolution volume, (b) Restored volume, (c) Mask function for spatial noise reduction, (d) Final high resolution volume.

where $\mathrm{G}_{\mathrm{i}}(\mathrm{w}), \mathrm{Fi}(\mathrm{w})$ and $\mathrm{H}(\mathrm{w})$ are frequency domain representation of $\mathrm{g}_{\mathrm{i}}(\mathrm{v}), \mathrm{f}_{\mathrm{i}}(\mathrm{v})$ and $\mathrm{h}(\mathrm{v})$, respectively. Here, $\mathrm{W}=(\mathrm{wx}$; wy; wz) denotes position of a voxel in 3D Fourier domain.We assume that the input noise is white and hence has a flat magnitude spectrum. So we get

$$
\left|G_{i}(\omega)\right|=N_{o}|H(\omega)|
$$

where, $\mathrm{N}_{\mathrm{o}}$ represents power of white noise. Therefore, the spectrum of the noise-only subvolume is the scaled version of the volume degradation function in the frequency domain. We assume that the volume degradation function is symmetric about the origin, therefore, it has zero phase response. For volume restoration, our main concern is the shape of the volume degradation function. So without any loss of generality, we can discard the scaling factor $\mathrm{N}_{0}$. Therefore, we can write

$$
\hat{H}_{i}(\omega)=\left|G_{i}(\omega)\right|
$$

where $\mathrm{H}_{\mathrm{i}}(\mathrm{W})$ represents the spectrum of the estimated degradation function from $\mathrm{i}^{\text {th }}$ noise-only block. The degradation function estimated from each block is then averaged. So, we obtain

$$
\hat{H}(\omega)=\frac{\sum_{i=1}^{M} \hat{H}_{i}(\omega)}{M}
$$


Finally we get the volume degradation function in the spatial domain by taking the inverse Discrete Fourier Transform (DFT) as follows

$$
\hat{h}(\mathbf{v})=\operatorname{IDFT}(\hat{H}(\omega)) .
$$

\subsection{Deconvolution}

We have implemented a two stage deconvolution scheme on recosntructed volume utilizing our estimated volume degradation function in order to reduce the affect of blurring of EM. The two stage deconvolution scheme consists of Richardson-Lucy (RL) followed by Wiener filtering deconvolution. RL ([14] [15]) method is an iterative expectation.

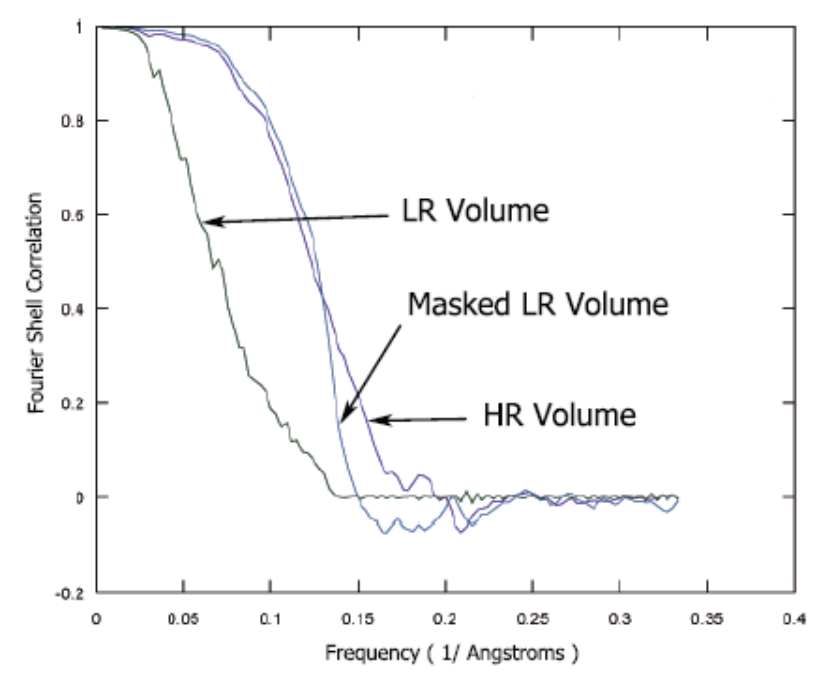

Fig. 5: FSC curve for molecule aaaa

maximization deconvolution algorithm based on the Bayesian framework. RL deconvolution has the advantage that it satisfies a priori volume-domain constraints (such as positivity, energy conservation, support constraints) automatically. Moreover, it has been reported that the beaminduced motion in cryo-EM is similar to the blurring caused in Hubble Telescope in astronomy [10]. Since RL produce satisfactory results in astronomy, it is expected that it will help in resolution enhancement of EM volume. Despite of the advantages, RL method has some disadvantages. The main shortcominngs are the noise amplifacation and spatially variable convergence rate. Wiener filtering is useful for suppressing noise and it is a conventional choice (for CTF correction) in cryo-EM [30] [31]. It has been reported that RL and Wiener filtering chain can be used to restore local information in image domain [32]. Therefore we have applied the Wiener filter after the RL deconvolution.

The update equation of the RL algorithm can be expressed as

$$
\begin{aligned}
& \hat{f}^{(r+1)}(\mathbf{v})=\hat{f}^{(r)}(\mathbf{v}) \\
& \times\left(\frac{g(\mathbf{v})}{\hat{f}^{(r)}(\mathbf{v}) * \hat{h}(\mathbf{v})} * \hat{h}(-\mathbf{v})\right)
\end{aligned}
$$


Where $\hat{f}^{(r)}(\mathrm{V})$ denotes the estimated volume at $\mathrm{r}^{\text {th }}$ iteration. We denote the last iteration of deconvolved volume using the RL algorithm as $\hat{f}^{R L}(\mathrm{v})$ Then the Wiener filter is used to estimate our final HR volume as

$$
\begin{gathered}
\hat{F}(\omega)=\left[\frac{1}{|\hat{H}(\omega)|} \times \frac{|\hat{H}(\omega)|^{2}}{|\hat{H}(\omega)|^{2}+K}\right] \hat{F}^{R L}(\omega) \\
\hat{f}(\mathbf{v})=\operatorname{IDFT}(\hat{F}(\omega))
\end{gathered}
$$

Here, $\hat{F}^{R L}(\omega)$ represents $\hat{f}^{R L}(\mathbf{v})$ in Fourier space, $\mathrm{K}$ is a specified constant according to SNR $\hat{H}(\omega)$ represents $\hat{h}(\mathbf{v})$ in Fourier space and $\hat{f}(\mathbf{v})$ is obtained deblurred volume which is the inverse Fourier Transform of $\hat{F}(\omega)$ In our work, the value of $\mathrm{K}$ is estimated as the ratio of noise energy and degraded LR volume's energy.

\subsection{Post Processing for Spatial Noise Reduction}

As stated earlier, the EM volume contains high level of noise outside the main molecular structure. This significantly deteriorates the volume quality at the boundary region. In many software package (i.e. XMIPP), user can manually form the mask in order to reduce the noise outside of macromolecule. In order to reduce this spatial noise, we incorporate an automatic spatial noise reduction technique. First, we calculate a binary mask function, m(v), by

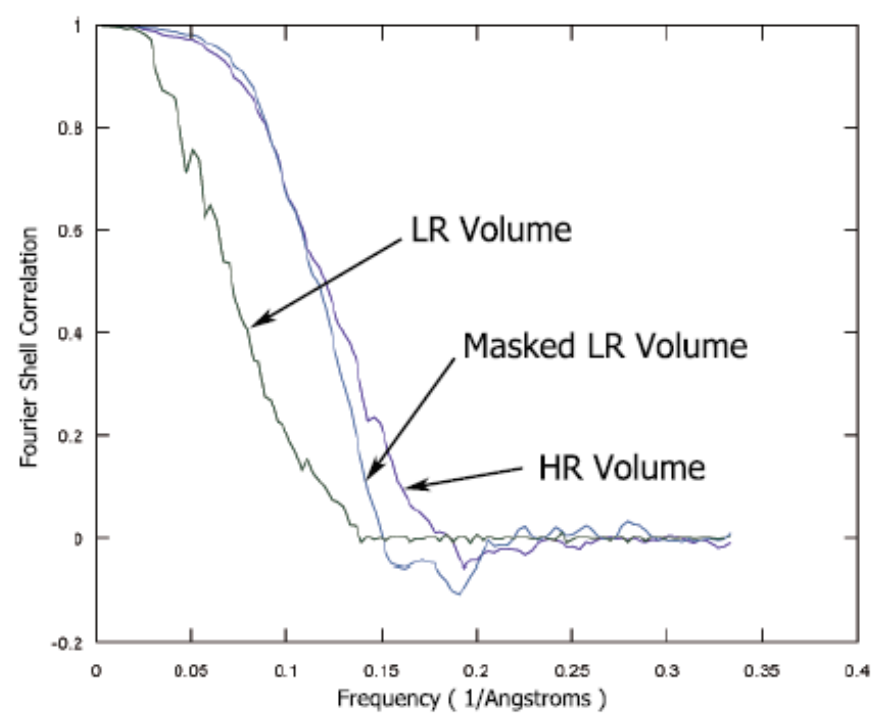

Fig. 6: FSC curve for molecule bbbb

thresholding the low resolution volume $\mathrm{g}(\mathrm{v})$. The threshold value, $\mathrm{t}$, is found using Otsu's method [33] [34]. The generation of the binary mask can be expressed as 


$$
m(\mathbf{v})= \begin{cases}1 & \text { if } g(\mathbf{v}) \geq t \\ 0 & \text { otherwise }\end{cases}
$$

As the pixel value is higher inside the molecular structure, most of the nonzero values in the mask function will indicate the structural elements. Moreover, the molecular structure is not disjoint and hence it will generate large clusters of 1's. On the other hand, the clusters of 1's due to noise will be disjoint and small in size. Therefore, we can easily remove these clusters using different morphological operations (erosion and dilation) [35]. In practice, $\mathrm{m}(\mathrm{v})$, is first eroded few times and then dilated to remove the clusters of 1's outside of the main structure. So after erosion and dilation, binary mask $\mathrm{m} 2(\mathrm{v})$ can be expressed as

$$
\begin{aligned}
& m_{1}(\mathbf{v})=m(\mathbf{v}) \ominus e(\mathbf{v}) \ominus e(\mathbf{v}) \ldots . . \ominus e(\mathbf{v}) \\
& m_{2}(\mathbf{v})=m_{1}(\mathbf{v}) \oplus d(\mathbf{v}) \oplus d(\mathbf{v}) \ldots . . \oplus d(\mathbf{v})
\end{aligned}
$$

Here, $\ominus, \oplus$ denote erosion and dilation operations, respectively and $\mathrm{e}(\mathrm{v})$ and $\mathrm{d}(\mathrm{v})$ are structure elements defined as $3 * 3 * 3$ cube of 1 . The number of erosion and dilation operation is performed depending on the SNR of the observed volume. In order to reduce the sharp edge, $\mathrm{m} 2(\mathrm{v})$ is convolved with a $3 \mathrm{D}$ gaussian shaped function $\mathrm{p}(\mathrm{v})$. So we get the final mask, $\mathrm{m} 3(\mathrm{v})$, as

$$
m_{3}(\mathbf{v})=m_{2}(\mathbf{v}) * p(\mathbf{v})
$$

The mask is multiplied with the deconvolved volume to generate the high resolution denoised volume. So we get

$$
f_{\text {est }}(\mathbf{v})=m_{3}(\mathbf{v}) \times \hat{f}(\mathbf{v})
$$

where $f_{\text {est }}(v)$ is the final enhanced high resolution volume.

\section{Simulation Results}

In this section, we present simulation results to demonstrate the performance of the proposed technique to enhance the macromolecular volume reconstructed by EM workflow. For evaluation of resolution improvement, we compute the Fourier Shell Correlation (FSC) curve of a macromolecular volume and respective ground-truth volume. FSC evaluates normalized crosscorrelation coefficient between two 3D volumes of corresponding shells in Fourier space 


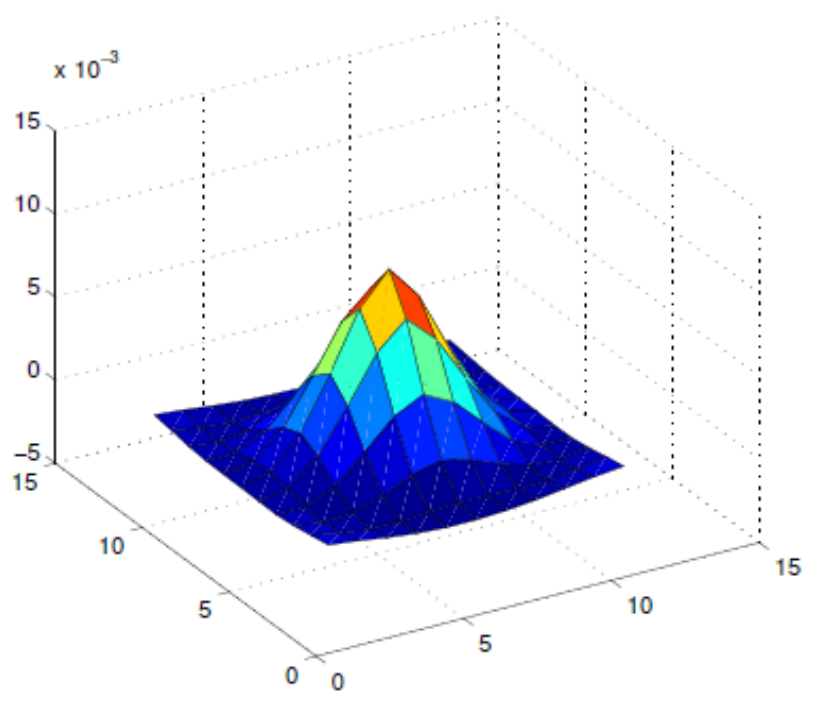

Fig. 7: Slice of estimated 3D volume degradation function of EMD-2605

[36] [37]. For evaluation of our technique, we compare the FSC curve of our enhanced volume with those of masked and unmasked observed volumes. We conducted experiment on two synthetic volumes and one real macromolecular volume. We have used the MATLAB functions deconvlucy and deconvwnr for regularized volume restoration using the estimated volume degradation function.

The synthetic degraded and noisy protein volumes( aaaa and bbbb of data size $220 * 220 * 220$ and $210 * 210 * 210$ respectively) are available in the website 3D Electron Microscopy Benchmark [38]. The procedure of generating these two synthetic volumes using Xmipp is described as follows. Two atomic structures were downloaded from PDB. Using xmipp volume from pdb at 1 Angstrom/pixel, these two structures were converted into voxel gray matrix. Then 50,000 random projections were generated for both images which were followed by adding white noise (with signal to noise ratio 0.1) and 34 contrast transfer functions (CTFs)( using parameters acceleration voltage $300 \mathrm{kV}$, defocus range $1.8-2.1 \mu \mathrm{m}$ and spherical aberration 2:26 mm) using xmipp phatom simulate microscope. We note that the CTFs were the same that had been used for Bovine Papilloma Virus [39]. For each molecule 10,000 projections were randomly selected and for each one of them we applied projection matching and CTF correction [40]. The resulting volumes were low pass filtered to the resolution approximated by the protocol described in this article [40]. Then these volumes can be assumed to be the resulting volume from cryo-EM workflow. Fig. 4 presents the $2 \mathrm{D}$ slice of two volumes at the different stages of our resolution enhancement algorithm. The leftmost slice represents the observed LR volume, slice after that shows the result of deconvolution step, slice after that represents the mask generated by our post processing technique and the right most slice represents our restored HR volume. We observe that our algorithm visually improves the resolution of both volumes and reduces noise outside of main macromolecular volume.

For quantitative analysis, the final restored volume are compared to the same structure at 1 resolution using the Fourier Shell Correlation (FSC). FSC curves are shown in Fig. 5 and Fig. 6 for LR volumes of aaaa and bbbb, respectively. It is observed that the FSC of our enhanced volume is better than that of LR volume. For comparison purpose, we also present the FSC curve of a masked LR volume (without any volume restoration), since masking is known to boost the FSC values. The masking boost the FSC in low frequency region only. In high frequency region, the FSC of the masked volune is significantly lower than that of our enhanced volume. 
We also implemented the proposed technique on an experimental EM volume EMD - 2605 [41] (of size $256 * 256 * 256$ ) obtained from [26]. A 2D slice of our estimated volume degradation function from EMD-2605 is shown in Fig. 7. It is apparent that the volume degradation function is spread over several points and so it will cause resolution loss. Fig. 8 presents 2D slices from the observed LR volume and enhanced HR volume. It is seen that the resolution of our restored volume has been improved by a significant amount and noise outside of main macromolecular volume is also reduced. Next, we present quantitative analysis of our resolution gain. As experimental EM volumes have no ground truth, one can use xmipp volume from pdb to generate ground-truth from atomic model [42]. We evaluated the
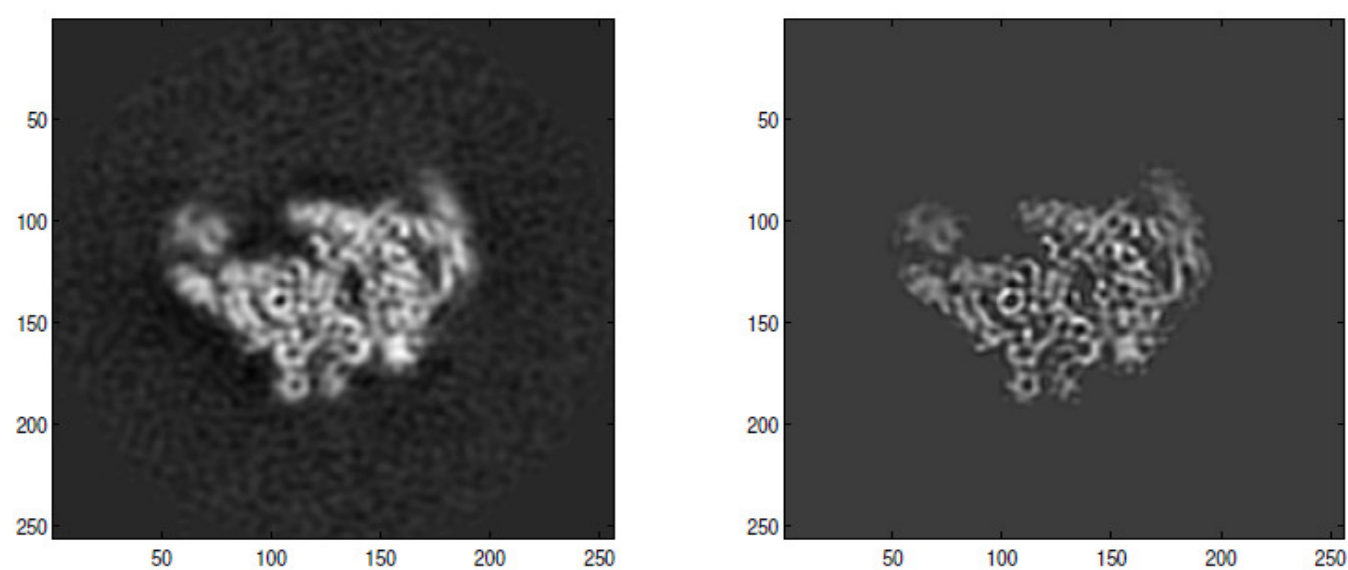

Fig. 8: Resolution enhancement of experimental volume EMD-2605. (Left) Low Resolution Volume. (Right) Estimated High Resolution Volume.

FSC of LR volume and reconstructed HR volume using this ground truth as reference volume. Fig. 9 presents the FSC curve corresponding to LR volume, HR volume and masked LR volume of EMD-2605. It is seen that our proposed technique significantly improves FSC at both low and high frequency region as compared to that of observed LR volume. To the contrary, masking of the observed LR volume only improves the low frequency region but fails in the high frequency region. As for estimating volume degradation function we have to average the noise-only subvolumes, the time complexity here will be linear to input volume. In each iteration, RL takes quadratic to input due to the convolution part. ForWeiner-filtering, we need to convert the volume to the frequency domain, so it takes quadratic of the input. So, the overall time complexity is quadratic.

\section{CONCLUSION}

Electron microscopy (EM) is a promising technique for macromolecular imaging that has several advantages over convnetional X-ray crystallography and NMR. However, the resolution of EM is rather low that can be improved significantly by applying signal processing methods after the 3D EM reconstruction workflow. We have proposed a novel technique for estimating the Volume Degradation Function from the observed low resolution EM volume and then used volume restoration techniques to enhance the resolution. Our technique also removes noise automatically outside of main macromolecular volume using some morphological operations. Simulation results confirmed the effectiveness of resolution enhancement by our proposed technique for both synthetic and experimental macromolecules. 


\section{CONFLICT OF INTEREST}

The authors declare that they have no conflict of interest.

\section{REFERENCES}

1. R.L. Marsden, T.A. Lewis, C.A. Orengo , "Towards a comprehensive structural coverage of completed genomes: a structural genomics viewpoint", BMC Bioinformatics, Vol. 8. Mar. 2007.

2. R. Henderson, "Realizing the potential of electron cryo-microscopy Quarterly Review of Biophysics", Cambridge University Press, Vol. 37, No.1, Jun. 2004, pp. 3-13.

3. X. Zhang, Z. H. Zhou, " Limiting factors in atomic resolution cryo electron microscopy: no simple tricks", J Struct Biol, Vol. 175, No. 1, Sep. 2011, pp. 253-263.

4. A.F. Brilot, J.Z. Chen, A. Cheng, J. Pan, S.C. Harrison, C.S. Potter, B. Carragher, R. Henderson, N. Grigorieff, "Beam-Induced Motion of Vitrified Specimen on Holey Carbon Film", J. Struct. Biol, Vol. 177, No. 3 , Mar. 2012, pp. 630-637.

5. M.G. Campbell, A. Cheng, A.F. Brilot, A. Moeller, D. Lyumkis, D. Veesler, J. Pan, S.C. Harrison, C.S. Potter, B. Carragher, Grigorieff, "Movies of ice-embedded particles enhance resolution in electron cryo-microscopy", Structure, Vol. 20, No.11, Nov. 2012, pp. 1823- 1828.

6. C.O. Sorzano , J. M. de la Rosa Trevín, J. Otón, J. J. Vega, J. Cuenca, A. Zaldívar-Peraza, J. GómezBlanco, J. Vargas, A. Quintana, R. Marabini and J.M. Carazo, "Semiautomatic, High-Throughput, High-Resolution Protocol for Three-Dimensional Reconstruction of Single Particles in Electron Microscopy ", Methods Mol Biol., vol. 950, 2013, pp. 171-193.

7. A.R. Faruqi and G. McMullan, ," Electronic detectors for electron microscopy", Q. Rev. Biophys., Vol. 44, No. 3, Aug. 2011, pp. 357-390.

8. G. McMullan, S. Chen, R. Henderson and A.R. Faruqi, “ Detective quantum efficiency of electron area detectors in electron microscopy", Ultrami- croscopy, Vol. 109, No. 9, Aug. 2009, pp. 11261143.

9. Y. Cheng, “Single-particle cryo-EM at crystallographic resolution", Cell, Vol. 161, No. 3, Apr. 2015, pp. 450-457.

10. W. Kühlbrandt, “The resolution revolution", Science, Vol. 343, No. 6178, Mar. 2014, pp. 14431444.

11. A. Bartesaghi, A. Merk, S. Banerjee, D. Matthies, X. Wu, J.L. Milne, S. Subramaniam , “ 2.2 resolution cryo-EM structure of galactosidase in complex with a cell-permeant inhibitor", Science , Vol. 348, No. 6239, Jun. 2015, pp. 1147-1151.

12 .K. Yonekura, S. Maki-Yonekura, K. Namba , "Complete atomic model of the bacterial flagellar filament by electron cryomicroscopy," Nature, Vol. 424, No. 6949, Aug. 2003, pp. 643-650.

13. R. Henderson, "Overview and future of single particle electron cryomicroscopy", Arch Biochem Biophys, Vol. 581, Sep 2015, pp. 19-24.

14. W.H. Richardson, "Bayesian-based iterative method of image restoration," JOSA, Vol. 62, No.1, 1972, pp. 55-59.

15. D.S.C. Biggs and M. Andrews "Acceleration of Iterative Image Restoration Algorithms," Applied Optics, Vol. 36. No. 8, 1997,pp. 1766-1775.

16. R.C. Gonzalez, R.E. Woods, "Digital Image Processing", Pearson Prentice Hall, 3rd ed., 2013, Ch. 5, pp. 311-389.

17. J. Frank "'Three-dimensional electron microscopy of macromolecular assemblies: visualization of biological molecules in their native state," Oxford University Press,2006.

18. Z.H. Zhou, "Towards atomic resolution structural determination by single-particle cryo-electron microscopy", Curr Opin Struct Biol, Vol. 18, No. 2, Apr. 2008, pp. 218-228.

19. S.H.W. Scheres, "RELION: Implementation of a Bayesian approach to cryo-EM structure determination", J. Struct. Biol, Vol. 180, No. 3, Dec 2012, pp. 519-530.

20. D. Lyumkis, A.F. Brilot, D.L. Theobald, N. Grigorieff, "Likelihood-based classification of cryo-EM images using FREALIGN', J. Struct. Biol., Vol. 183, No. 3, Sep 2013 , pp. 377-388.

21. C.O. Sorzano, J.R. Bilbao-Castro, Y. Shkolnisky, M. Alcorlo, R. Melero, G. Caffarena-Fernández , M. Li , G. Xu, R. Marabini , J.M. Carazo, “ A clustering approach to multireference alignment of single-particle projections in electron microscopy", J Struct Biol, Vol. 171, No. 2, Aug. 2010, pp. 197-206 . 
22. S.H.W. Scheres , M. Valle, R. Núñez, C.O. Sorzano, R. Marabini, G.T. Herman, J.M. Carazo ,“ Maximum-likelihood multi-reference refinement for electron microscopy images", J Mol Biol, Vol. 348, No. 1, Apr. 2005, pp. 139-149.

23. A. Pascual-Montano , L.E. Donate, M. Valle, M. Bárcena , R.D. Pascual-Marqui , J.M. Carazo , "A novel neural network tecnique for analysis and classification of EM single-particle images", J Struct Biol, Vol. 133, No. 2-3, Feb-Mar 2001, pp. 233-245.

24. R.A. Crowther, L.A. Amos , "Harmonic analysis of electron microscope images with rotational symmetry", J Mol Biol, Vol. 60, No. 1, Aug 1971, pp. 123-130 .

25. P.A. Penczek, J. Zhu, R. Schroder, J. Frank, “ Three dimensional reconstruction with constrast transfer compensation from defocus series", Scanning Microscopy, Vol.11, 1997, pp. 147-154.

26. https://pdbj.org/emnavi/quick.php?id=emdb-2605

27. I. Sabanay, T. Arad, S. Weiner, B. Geiger, "Study of vitrified, unstained frozen tissue sections by cryoimmunoelectron microscopy," J Cell Sci, Vol. 100, Sep. 1991, pp. 227-236.

28. J. Dubochet, M. Adrian , J.J. Chang, J.C. Homo, J. Lepault, A.W. McDowall, P. Schulz "“ Cryoelectron microscopy of vitrified specimens," Q Rev Biophys, Vol. 21, No.2, May 1988, pp. 129-228.

29. J.L. Leunissen and H. Yi ,"Self-pressurized rapid freezing (SPRF): a novel cryofixation method for specimen preparation in electron microscopy", J Microsc, Vol. 235, No. 1, Jul. 2009, pp. 25-35.

30. C.V. Sindelar and N. Grigorieff, "An adaptation of the Wiener filter suitable for analyzing images of isolated single particles", J Struct Biol., Vol. 176, No. 1, Oct. 2011, pp. 60-74.

31. P.A. Penczek, "Image Restoration in Cryo-electron Microscopy", Methods Enzymol., vol. 482, 2010, pp. $35-72$

32. F. Tsumuraya , "Deconvolution based on the Wiener-Lucy chain algorithm: an approach to recover local information losses in the deconvolution procedure ",JOSA, Vol. 13, No. 7, 1996, pp. 1532-1536.

33. N. Otsu, "A threshold selection method from gray-level histograms", IEEE Trans. Sys. Man. Cyber., Vol. 9, No. 1, Jan 1979,pp. 62-66.

34. M. Sezgin and B. Sankur, "Survey over image thresholding techniques and quantitative performance evaluation," J. Electron. Imaging, Vol. 13, No. 1, Jan. 2004, pp. 146-165.

35. R.C. Gonzalez, R.E. Woods, "Digital Image Processing", Pearson Prentice Hall, 3rd ed., 2013, Ch. 9, pp. 311-389.

36. G. Harauz, M. van Heel, "Exact filters for general geometry three dimensional reconstruction", Optik, vol 73, No. 4, 1986, pp.146-156.

37. N. Grigorieff, "Resolution measurement in structures derived from single particles",Acta Crystallogr D Biol Crystallogr., Vol.56, No. 10, Oct. 2000, pp. 1270-1277.

38. 3D Electron Microscopy Benchmark. [Online]. Available: http://i2pc.cnb.csic.es/3dembenchmark/LoadAboutUs.htm

39. M. Wolf, R.L. Garcea, N. Grigorieff, S.C. Harrison, " Subunit interactions in bovine papillomavirus", Proc. Natl. Acad. Sci. USA, Vol. 107, No. 14, Apr. 2010, pp. 6298-6303.

40. S.H.W. Scheres, R. Núñez-Ramírez, C.O.S. Sorzano, J.M. Carazo , R. Marabini, "Image processing for electron microscopy single-particle analysis using XMIPP Nature Protocols", Vol. 3, 2008, pp. 977-990.

41. B. Feng, C.S. Mandava, Q. Guo, J. Wang, W. Cao, N. Li, Y. Zhang, Y. Zhang, Z. Wang, J. Wu, S. Sanyal, J. Lei, N. Gao , "Structural and functional insights into the mode of action of a universally conserved Obg GTPase",PLOS Biol, Vol. 12, No. 5. ,2014.

42. C.O.S. Sorzano, J. Vargas, J. Otón, V. Abrishami, J.M. de la Rosa-Trevín, S. del Riego, A. Fernández-Alderete, C. Martínez-Rey, R. Marabini, J.M. Carazo, "Fast and accurate conversion of atomic models into electron density maps",AIMS Biophysics, Vol. 2, No. 1, 2015, pp. 8-20.

43. Gupta, K. D., Ahsan, M., Andrei, S. (2018). Extending the Storage Capacity And Noise Reduction of a Faster QR-Code. BRAIN. Broad Research in Artificial Intelligence and Neuroscience, 9(1), 59-71.

44. Gupta, K. D., Sen, S. (2018). A Genetic Algorithm Approach to Regenerate Image from a Reduce Scaled Image Using Bit Data Count. BRAIN. Broad Research in Artificial Intelligence and Neuroscience, 9(2), 34-44.threshold selection method from gray-level histograms", IEEE Trans. Sys. Man. Cyber., Vol. 9, No. 1, Jan 1979, pp. 62-66. 